\title{
The Effect of Gallic Acid on Motor Learning and Cerebellar Level of Brain Derived Neurotrophic Factor in a Rat Model of Autism
}

\author{
Mohammad Amin Edalatmanesh*, Parvin Samimi
}

Department of Physiology, College of Sciences, Shiraz Branch, Islamic Azad University, Shiraz, Iran

Article Info:

\section{A BSTRACT}

Introduction: Exposure to Valproic acid (VPA) during pregnancy in rats leads to oxidative stress, brain deficits, and autistic-like behaviors in the offspring. On the other hand, Gallic acid (GA) can effectively be used in the treatment of free radicals-induced nervous system disorders. The aim of this study was to investigate the effect of GA on motor learning and cerebellar level of brain derived neurotrophic factor (BDNF) in rats embryonically exposed to VPA. Materials and Methods: Pups of 30 pregnant rats were randomly divided into 5 groups: control, VPA (500 mg/kg Valproic acid) at embryonic day (ED) 12.5 groups as well as three groups of VPA+GA50, VPA+GA100, and VPA+ GA200 (receiving 50, 100 and $200 \mathrm{mg} / \mathrm{kg}$ GA, respectively). GA was orally administered from 12 to $19 \mathrm{ED}$. Then, to assess the motor disorders beam walking and rotarod tests were carried out at postnatal day 30. Finally, the cerebellar level of BDNF was measured using ELISA. Results: Impairments were observed in the motor function test of the VPA group compared to the control rats. In contrast, GA-treated groups have a significant improvement in speed on beam and balance in beam walking test as well as an increase time spent in rotarod as well. A significant increase of BDNF was seen in GA-treated rats compared to VPA group. Conclusion: GA can alleviate the adverse effects of VPA on motor learning and balance in a rat model of autism.

\section{Key words:}

1. Gallic Acid

2. Valproic Acid

3. Motor Disorders

4. Autistic Disorder

5. Rats

*Corresponding Author: Mohammad Amin Edalatmanesh

E-mail: amin.edalatmanesh@gmail.com 


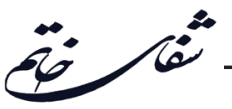

\title{
اثر كاليك اسيد بر يادَيرى حركتى و سطح فاكتور نوروتروفيك مشتق از مغز در مخجٌه موش هاى صحرايى مدل اوتيسم فور لوروفي
}

\author{
محمد امين عدالت منش"، يروين صميمى
}

كروه فيزيولوزى، دانشكده علوم، واحد شيراز، دانشكاه آزاد اسلامى، شيراز، ايران

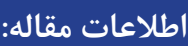

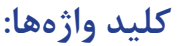

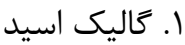

r. r. واليروئيك اسيد اليد

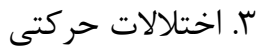

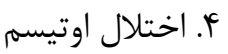

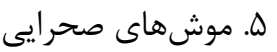

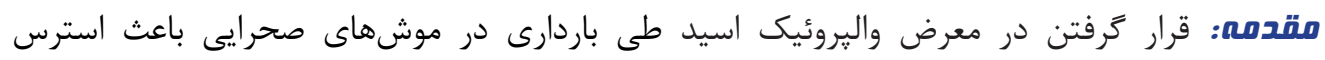

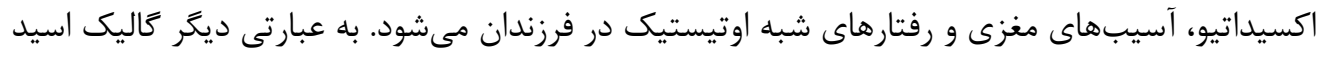

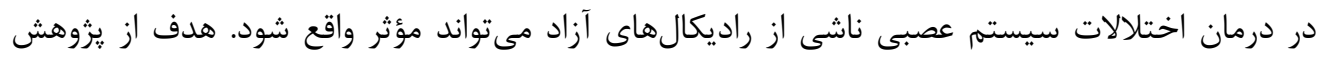

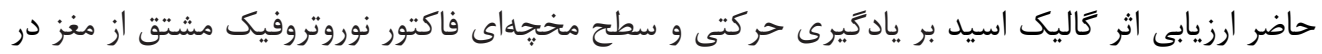

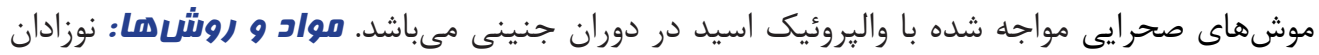

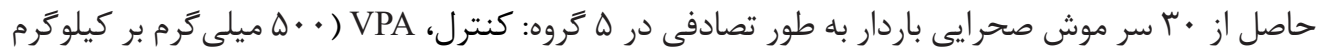

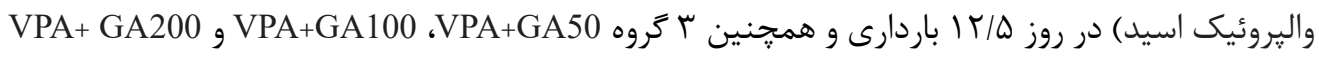

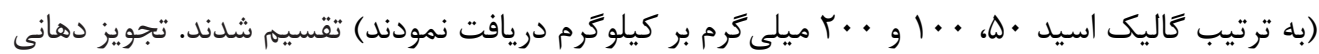

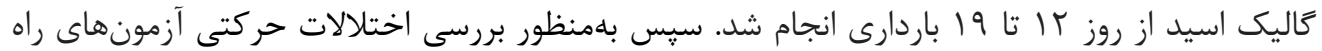

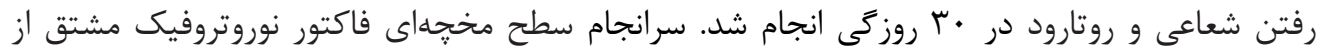

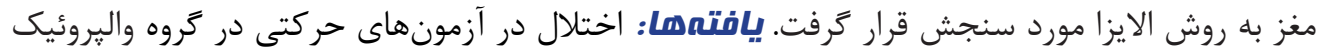

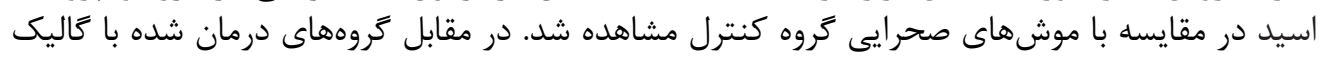

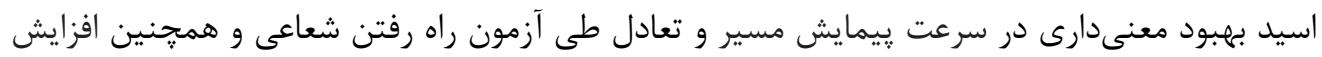

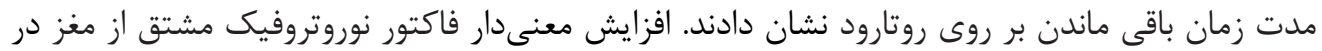

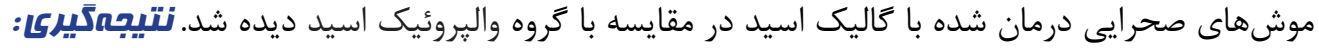

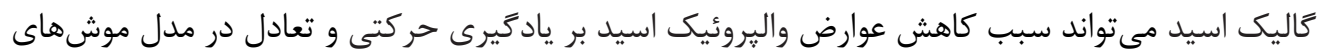
صحرايى اوتيسم شود.

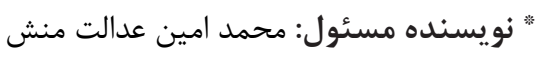
آدرس الكترونيكى: amin.edalatmanesh@gmail.com 


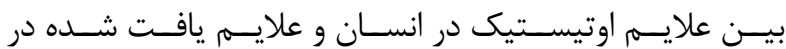

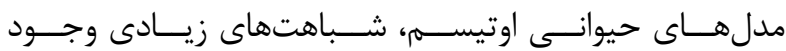

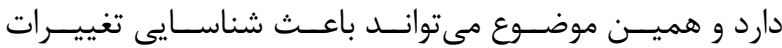

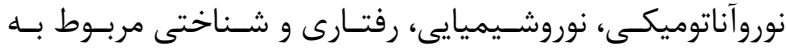

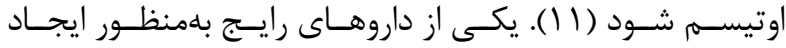

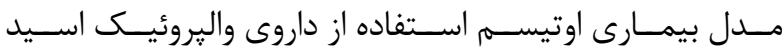
(VPA)

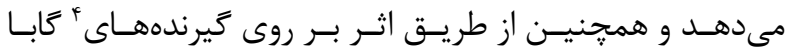

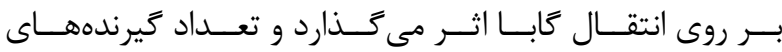

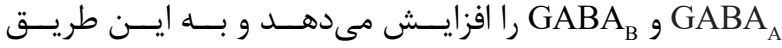

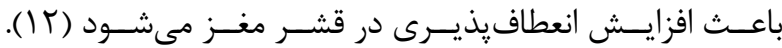

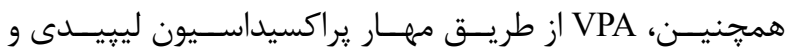

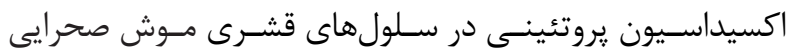

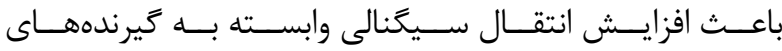
NMDA

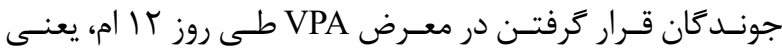

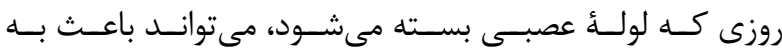

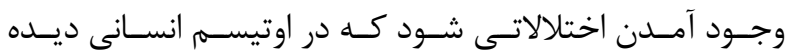

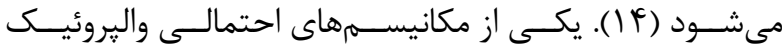

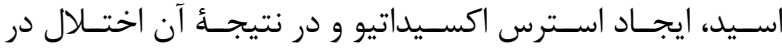

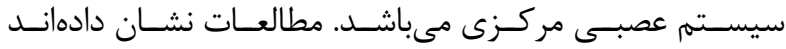

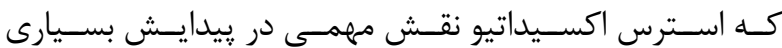

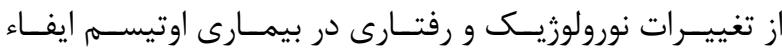

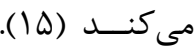

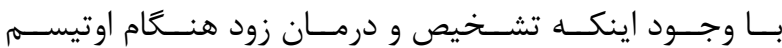

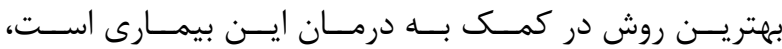

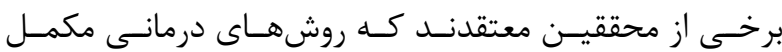

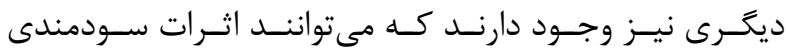

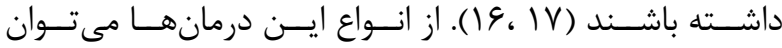

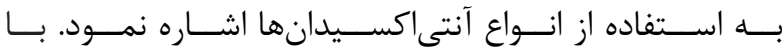

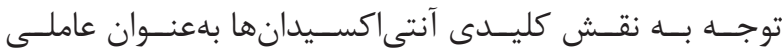

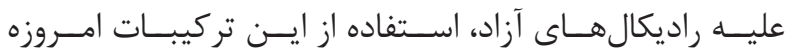

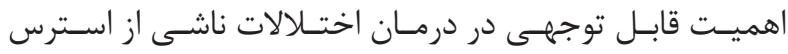

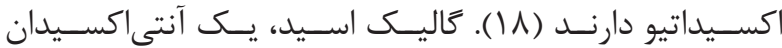

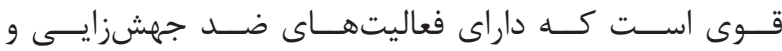

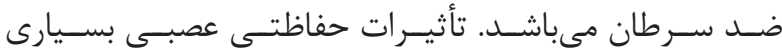

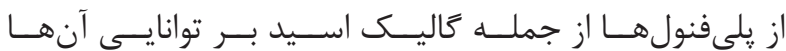

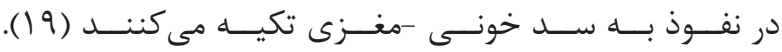

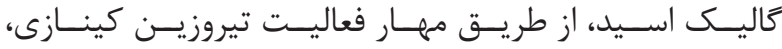

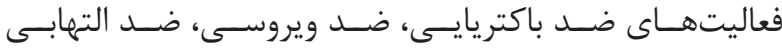

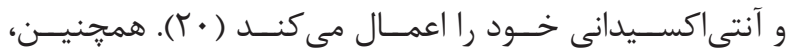

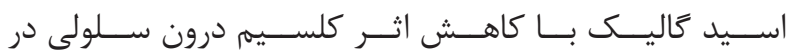

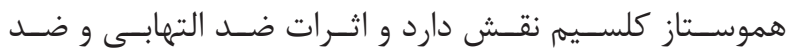

${ }^{1}$ Neurotransmitter

${ }^{2}$ Brain derived neurotrophic factor

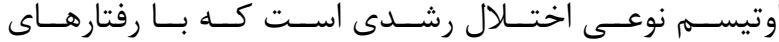

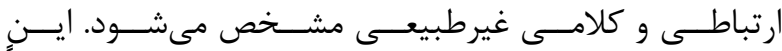

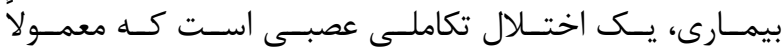

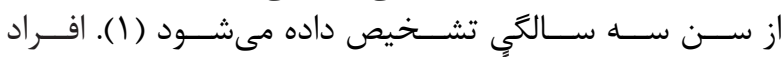

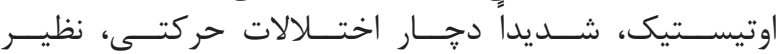

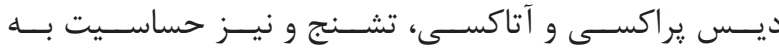

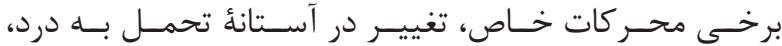

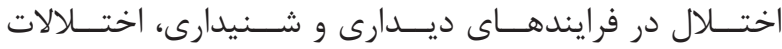

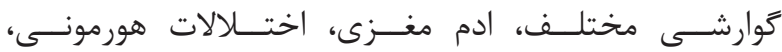

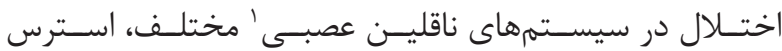

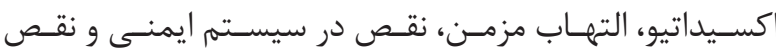

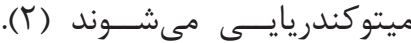

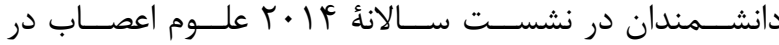

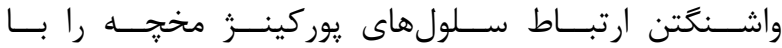

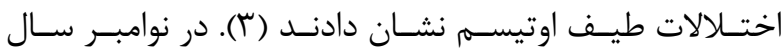

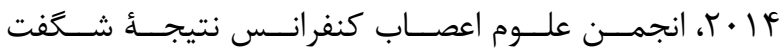

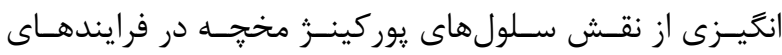

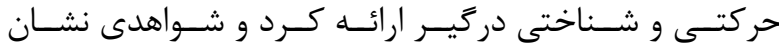

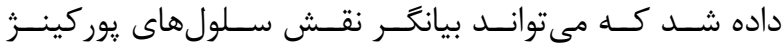

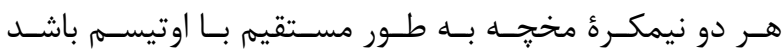

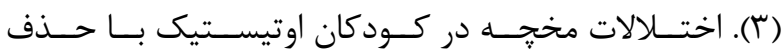

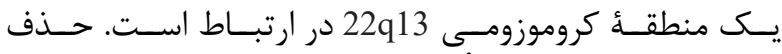

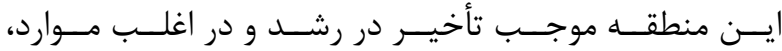

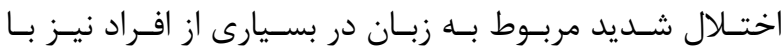

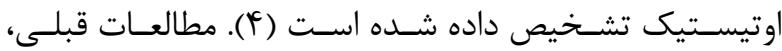

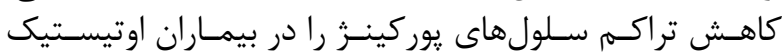

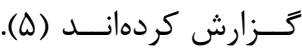

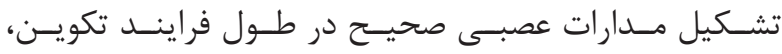

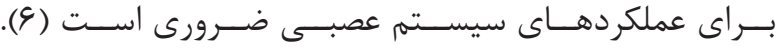

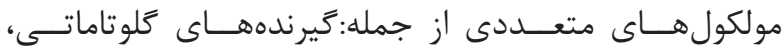

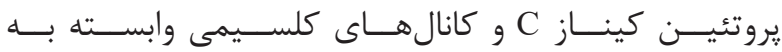

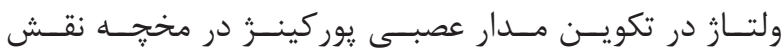

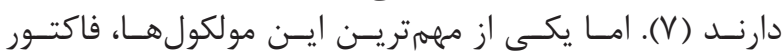

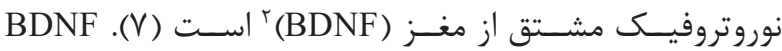

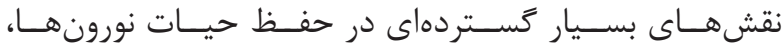

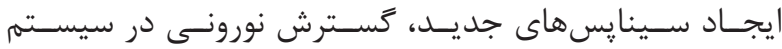

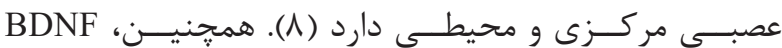

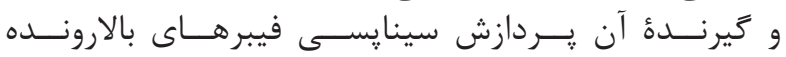

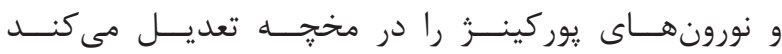

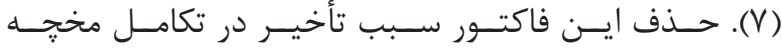

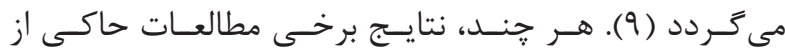

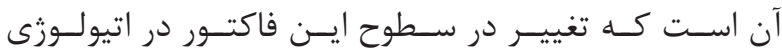

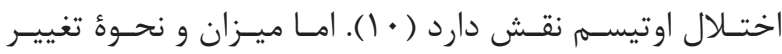

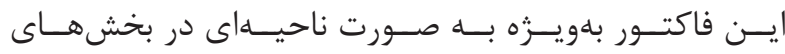

\footnotetext{
${ }^{3}$ Valproic acid

${ }^{4}$ Receptors
} 


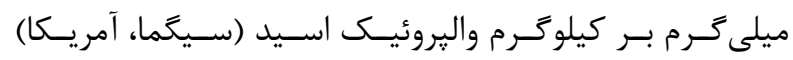

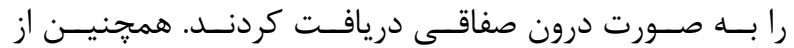

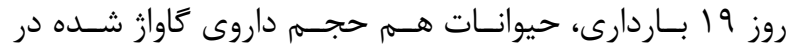

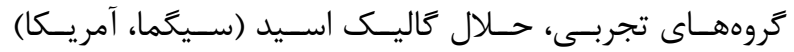

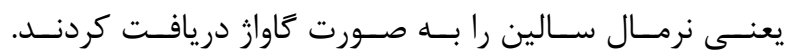

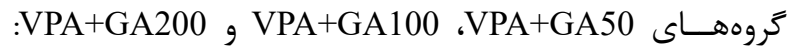

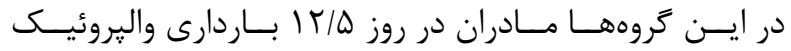

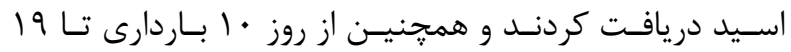

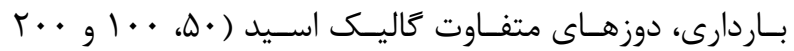

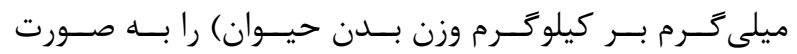

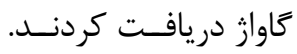

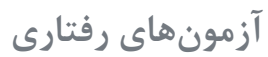

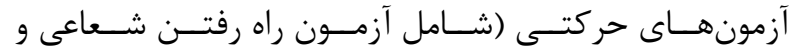

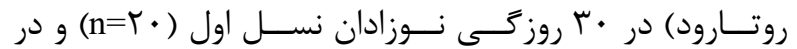

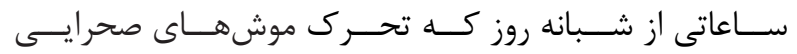

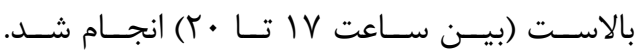

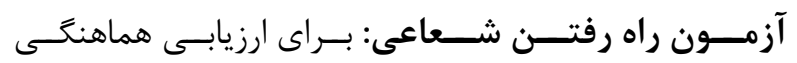

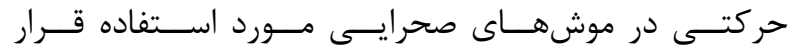

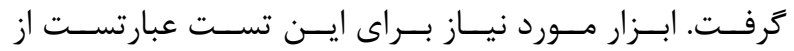

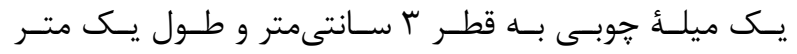

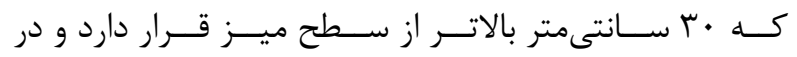

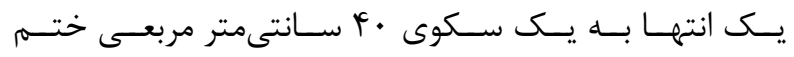

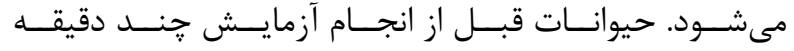

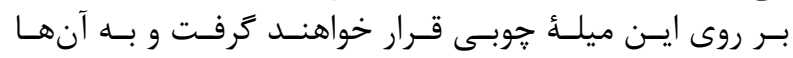

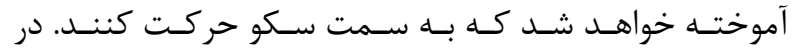

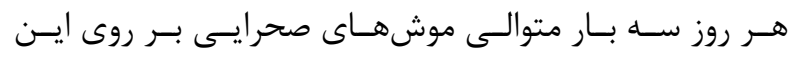

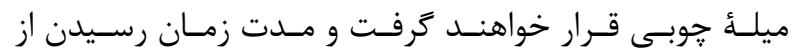

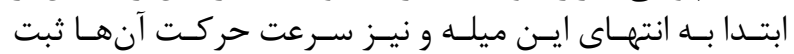

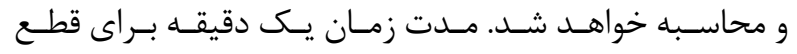

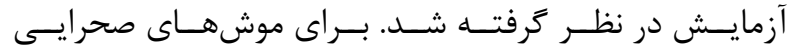

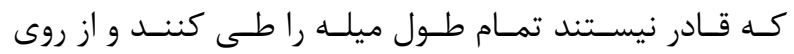

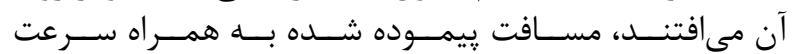

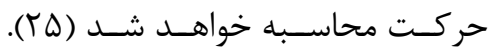

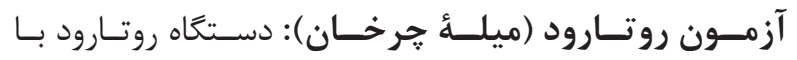

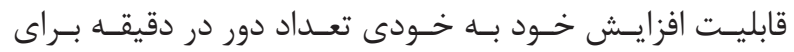

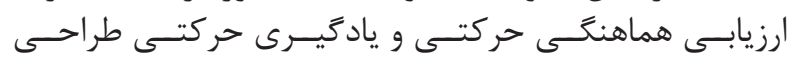

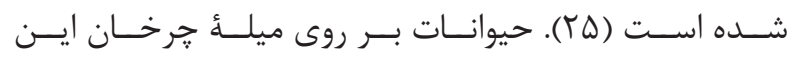

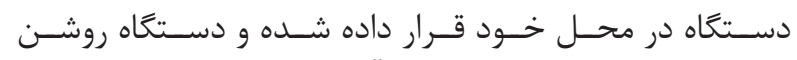

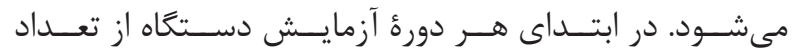

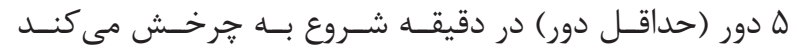

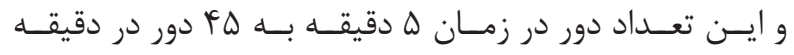

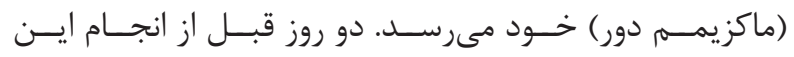

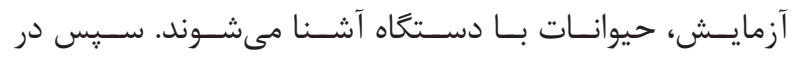

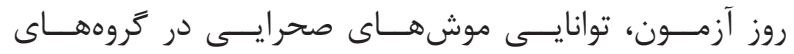

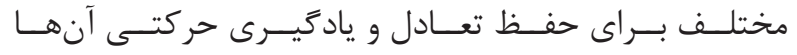

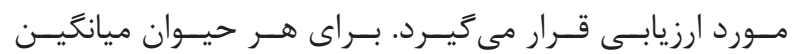

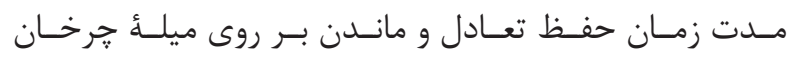

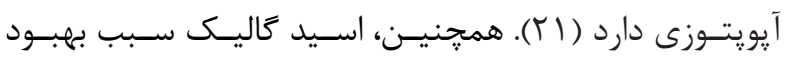

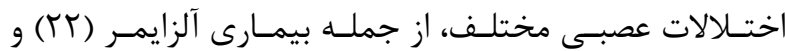

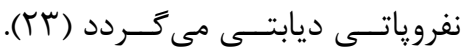

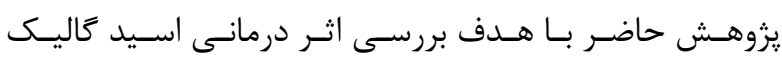

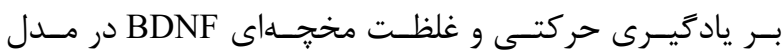

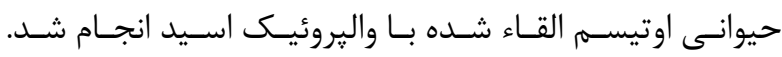

مواد و روشها

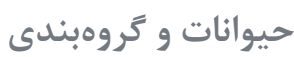

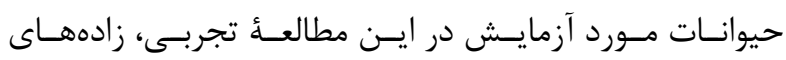

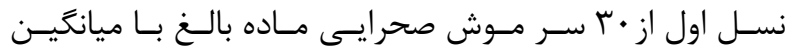

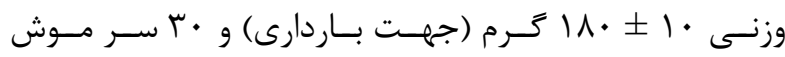

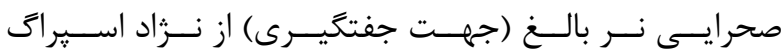

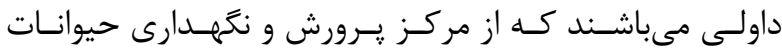

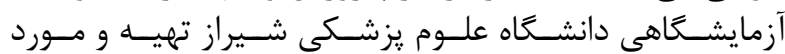

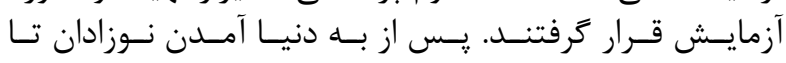

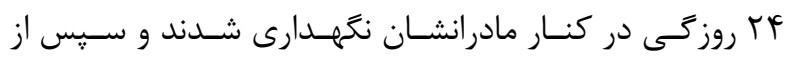

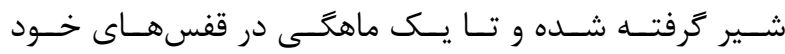

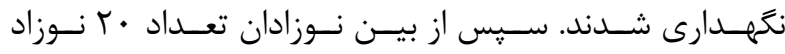

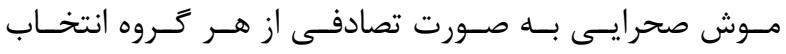

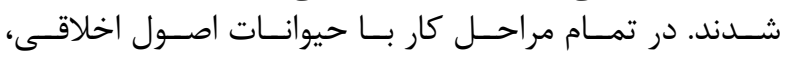

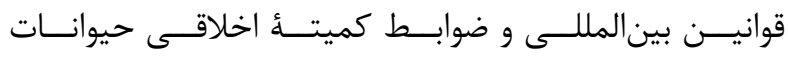

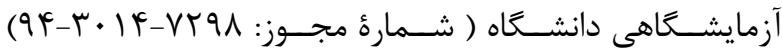

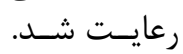

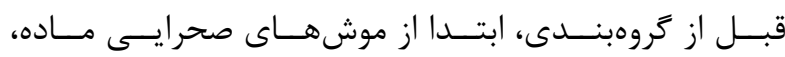

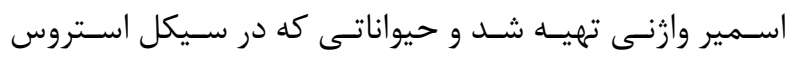

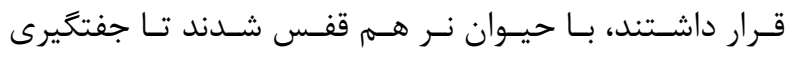

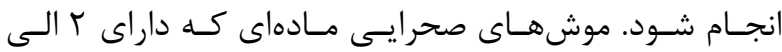

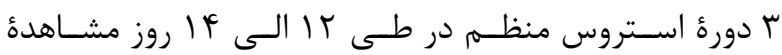

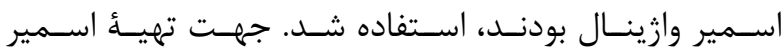

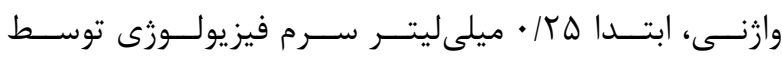

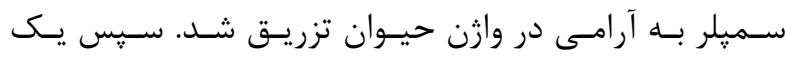

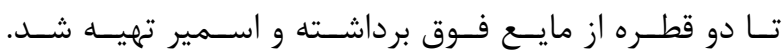

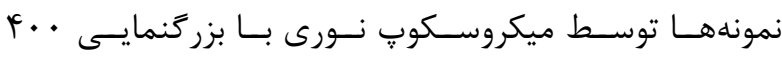

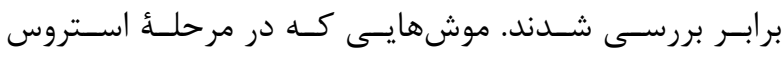

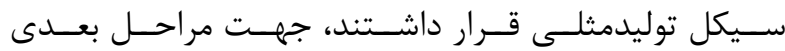

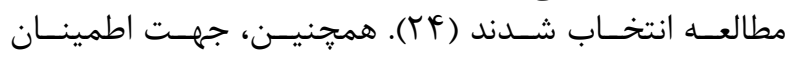

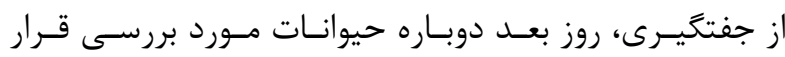

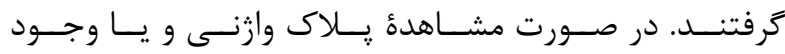

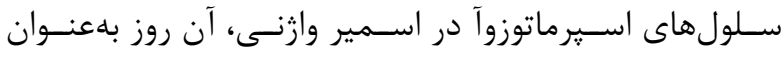

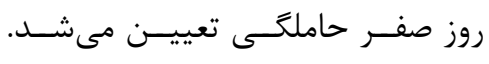

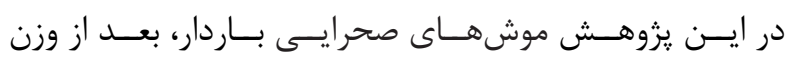

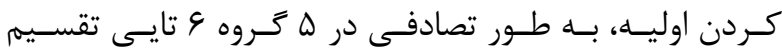

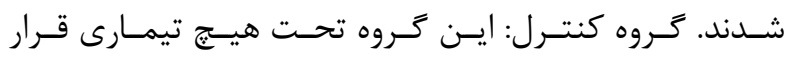

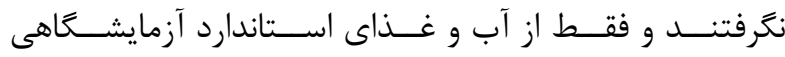

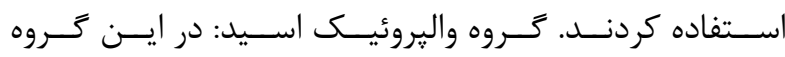

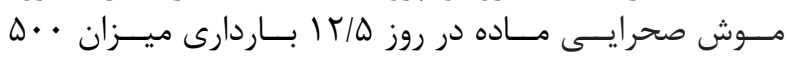




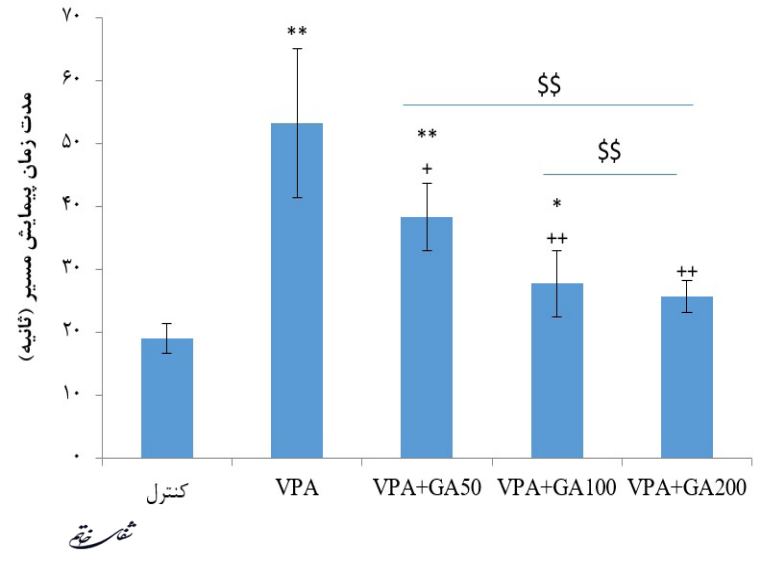

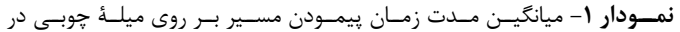

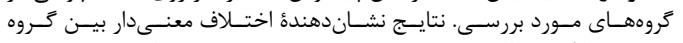

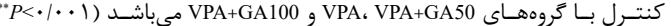

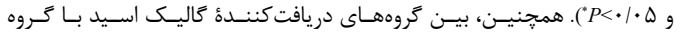

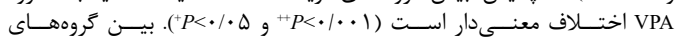

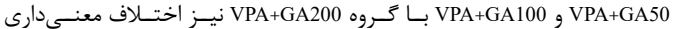

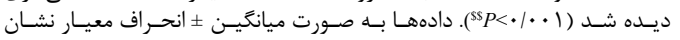

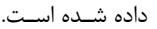

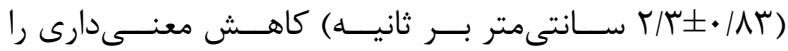

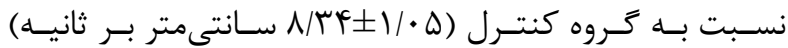

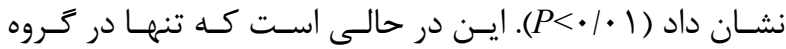

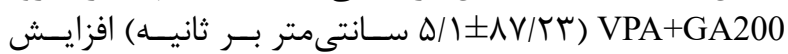

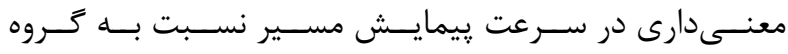

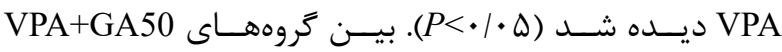

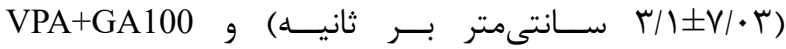

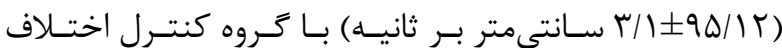

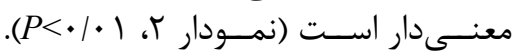

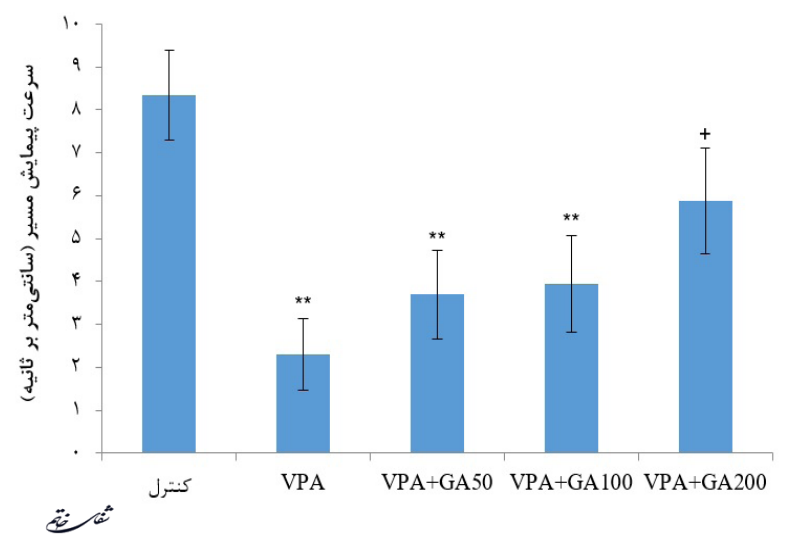

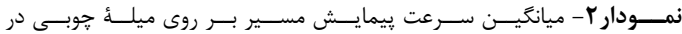

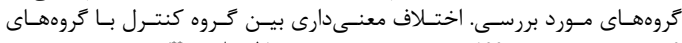

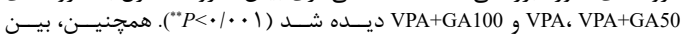

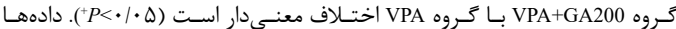

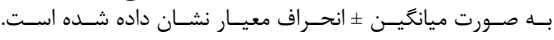

بررسى مدت زمان باقى ماندن بر روتارود

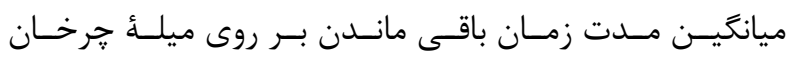

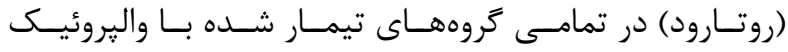

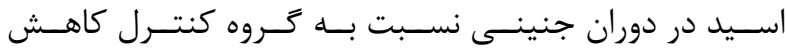

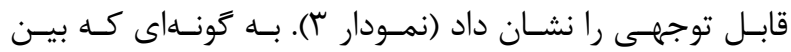

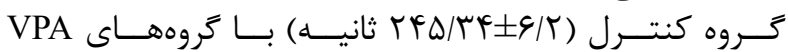

بــا حداقـل ســه تكـــار ثبـت شـد (†ه). سنجش سطح مخجهاى BDNF

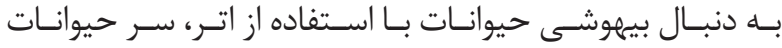

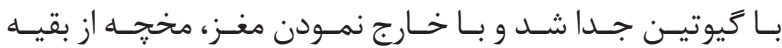

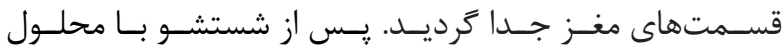

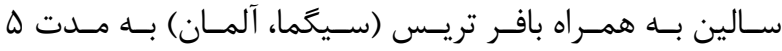

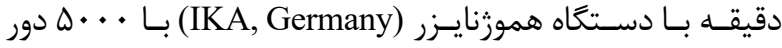

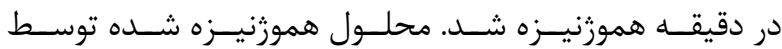

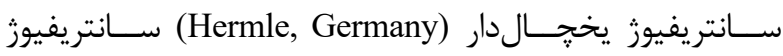

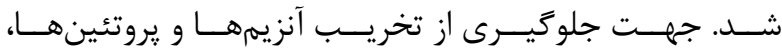

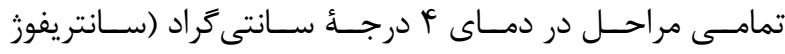

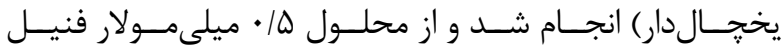

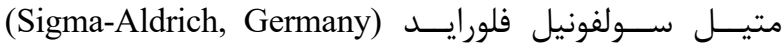

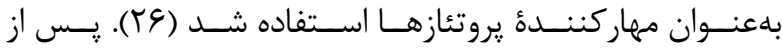

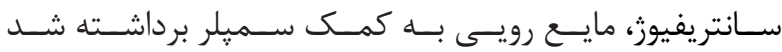

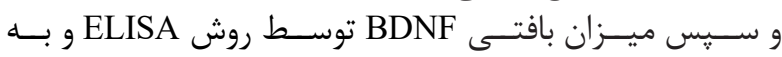

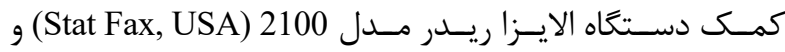

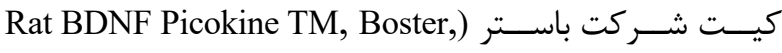

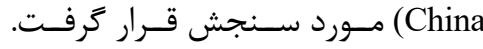
تحليل آمارى

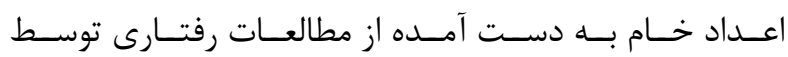

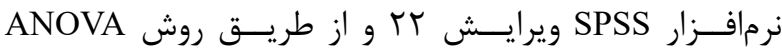

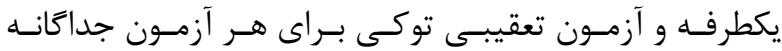

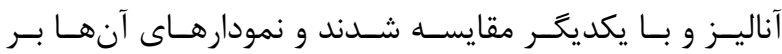

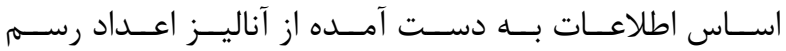

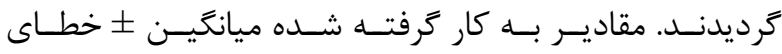

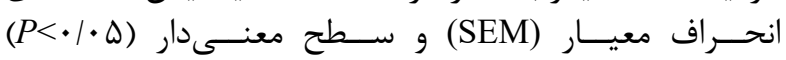
مى باشـــد. يافتهها

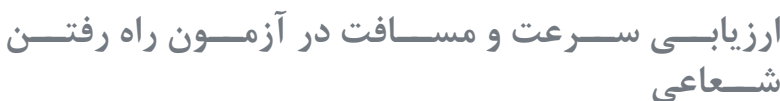

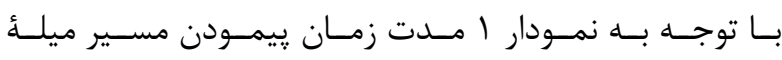

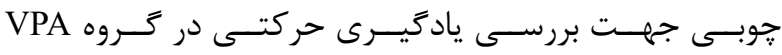

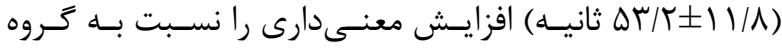
كنتــرل (T/

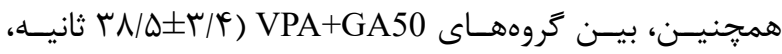

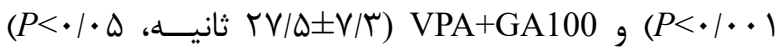

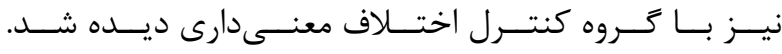

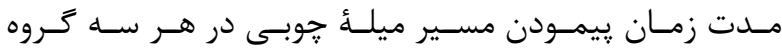

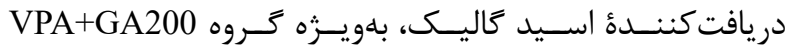
نش

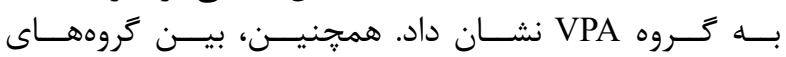

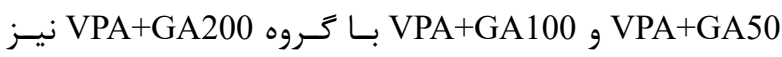

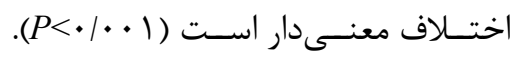

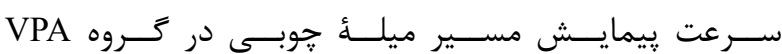


بحث و نتيجه كَيرى

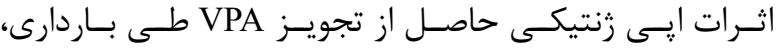

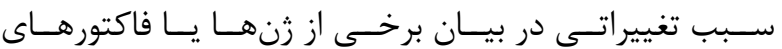

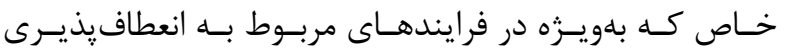

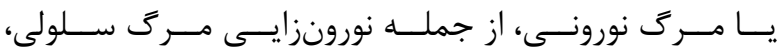

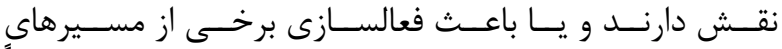

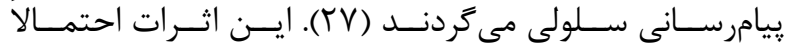

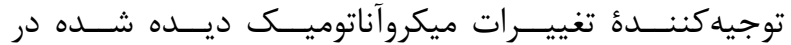

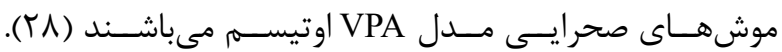

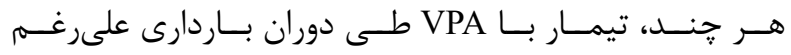

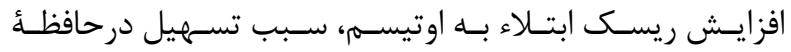

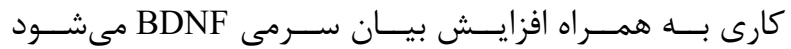

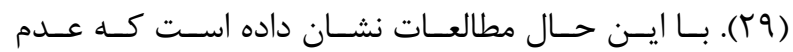

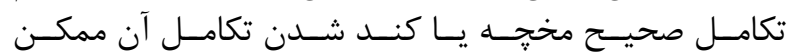

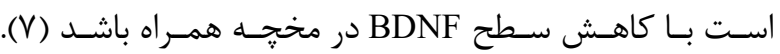

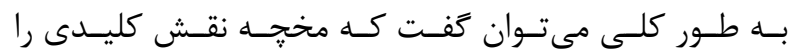

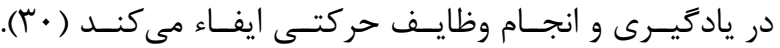

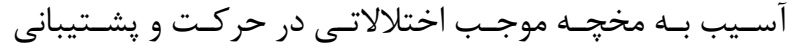

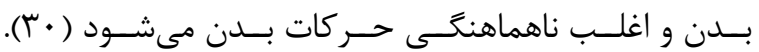

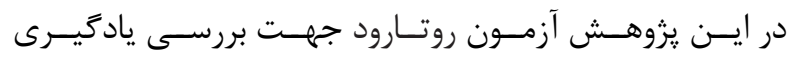

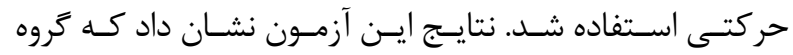

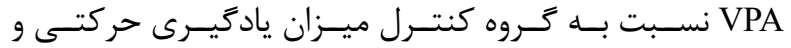

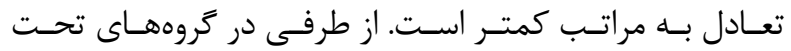

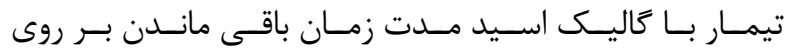

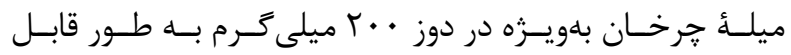

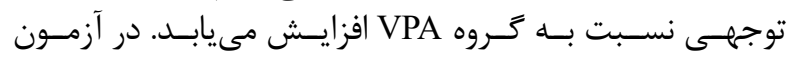

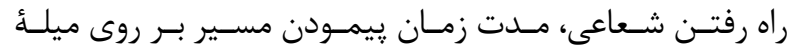

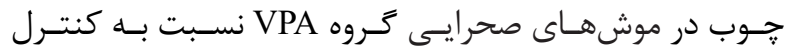

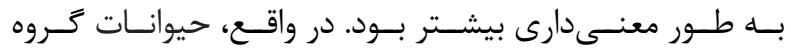

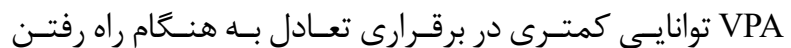

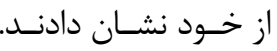

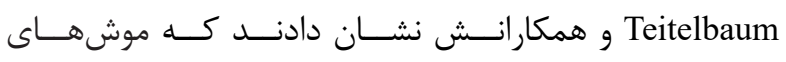

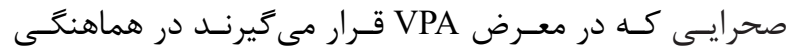

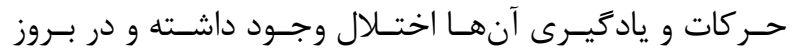

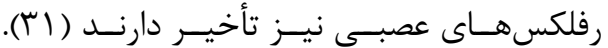

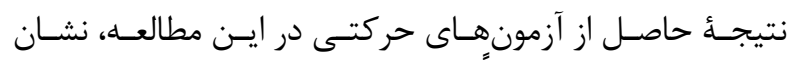

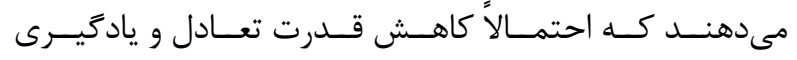

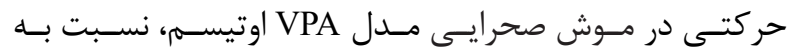

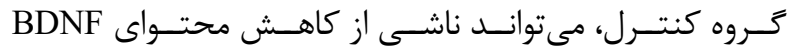

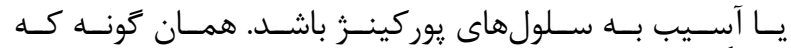

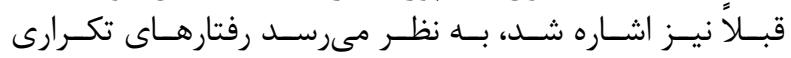

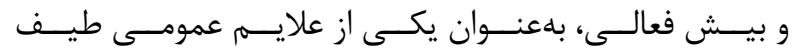

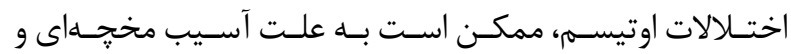

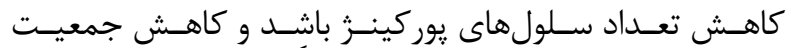

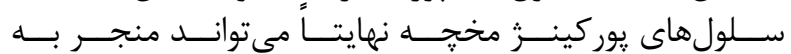

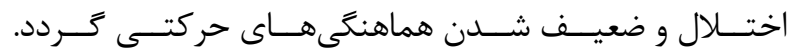

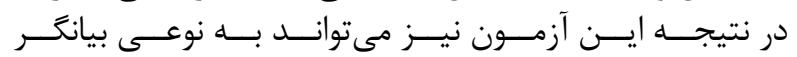

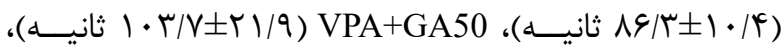

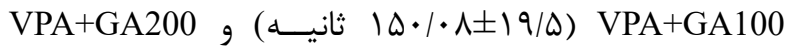

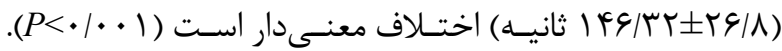

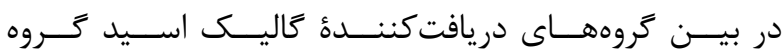

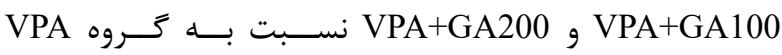

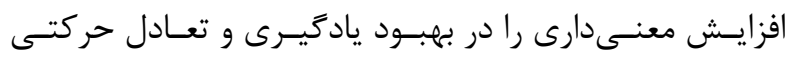

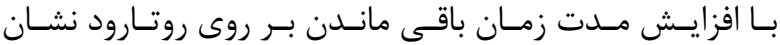
داد (P) (

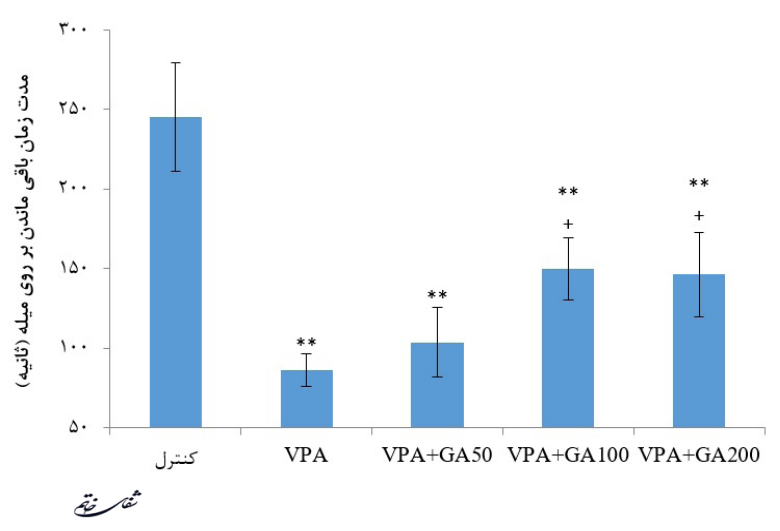

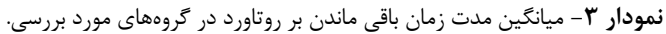

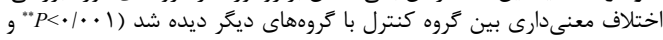

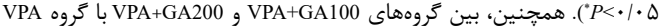

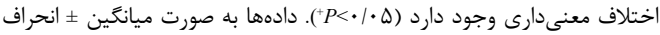

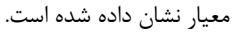

سنجش سطح مخجهای BDNF

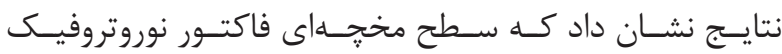

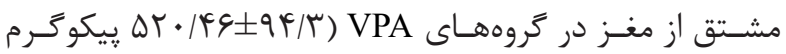

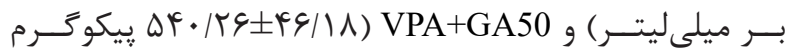

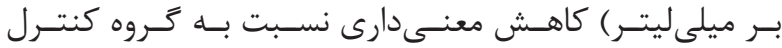

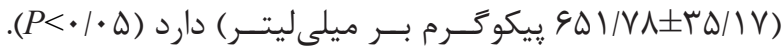

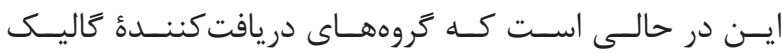

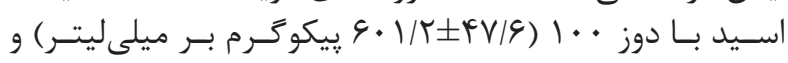

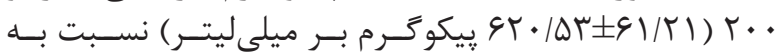

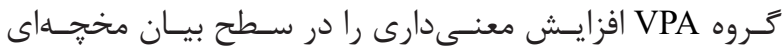

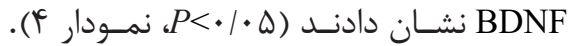

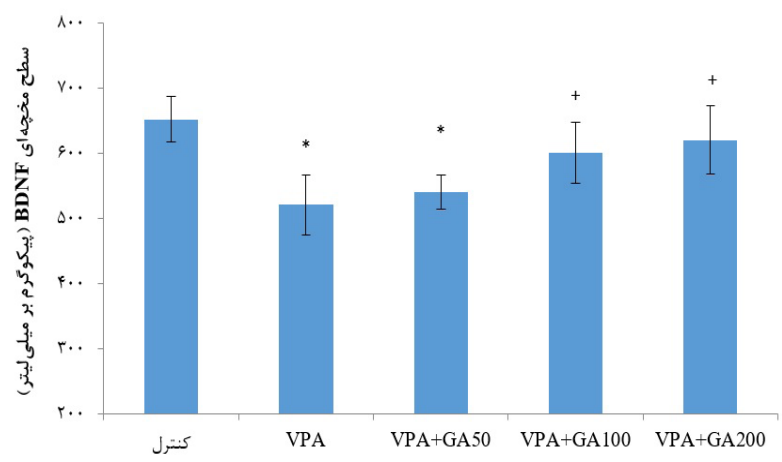
ثمث

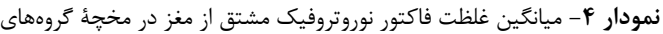

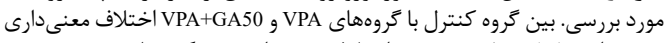

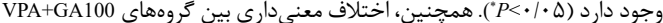

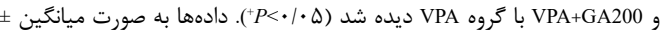
انحراف معيار نشان داده شده است. 


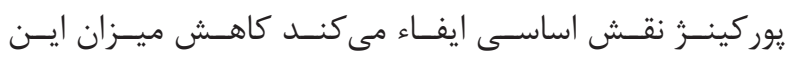

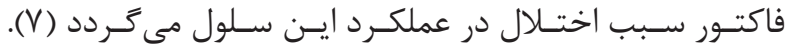

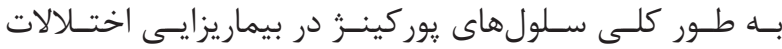

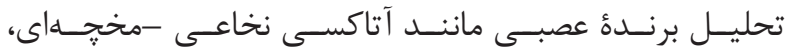

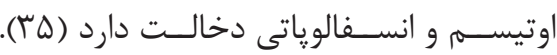

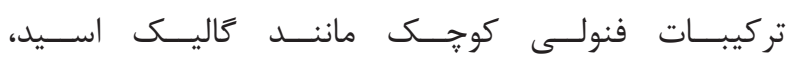

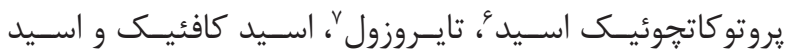

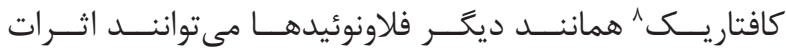

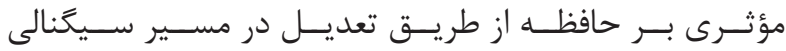

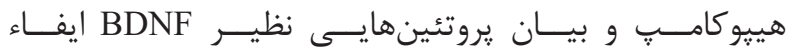

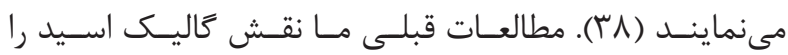

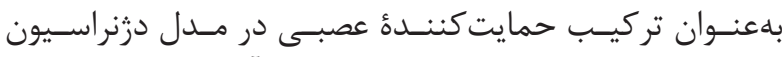

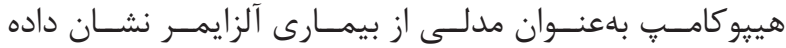

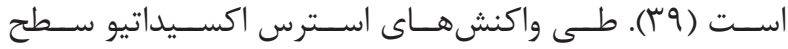

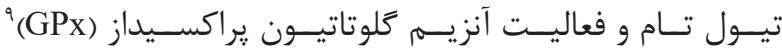

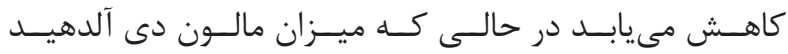

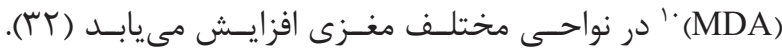

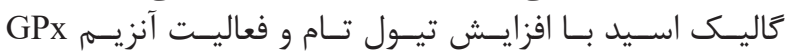

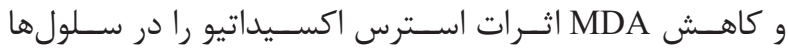

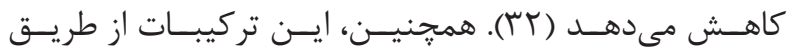

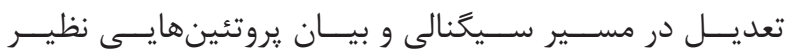

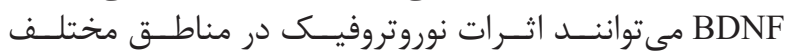

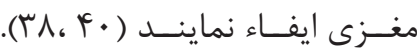

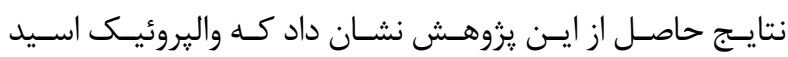

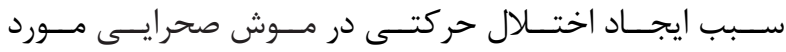

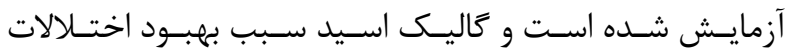

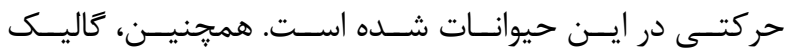

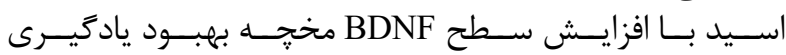

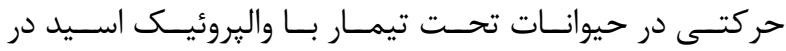

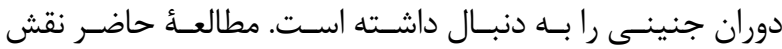

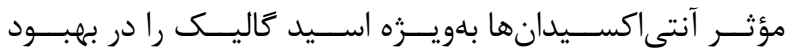
علايسم حركتـى در بيمـاران اوتيسـتيكى توصيـهـ مى كنــد.

$$
\text { تشكر و قدردانى حرنى }
$$

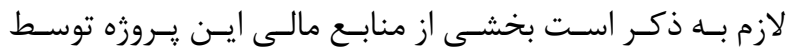

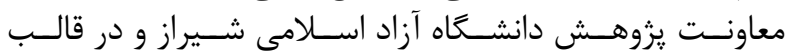

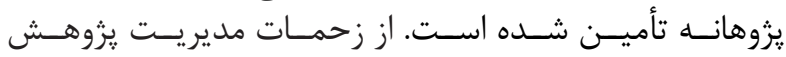

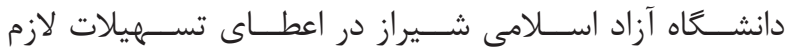

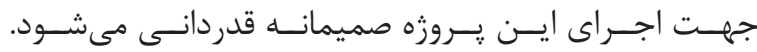

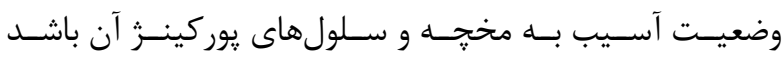

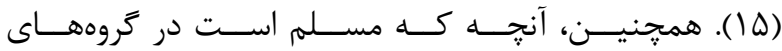

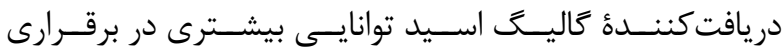

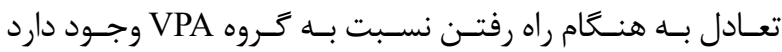

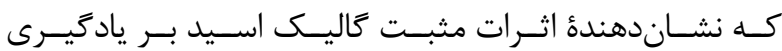

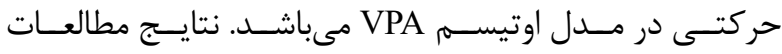

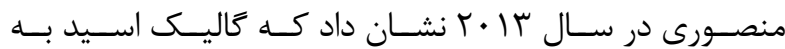

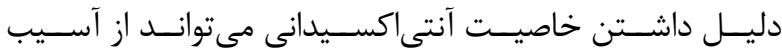

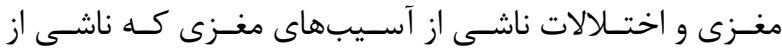

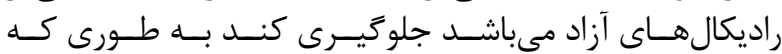

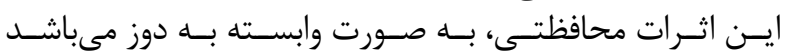

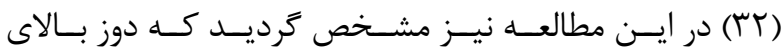

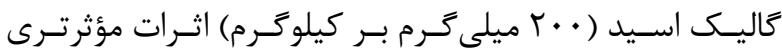

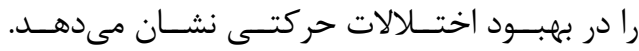

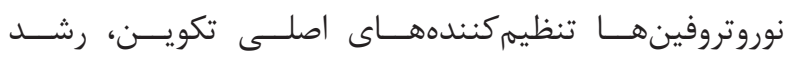

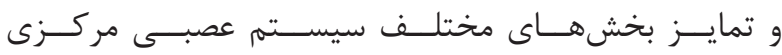

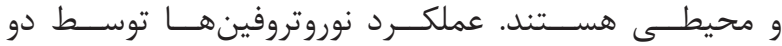

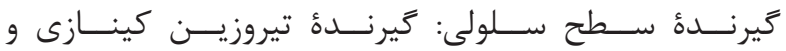

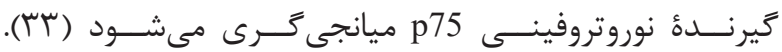

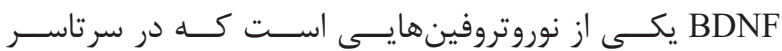

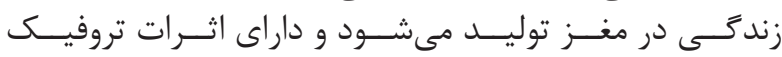

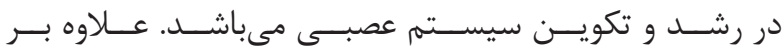

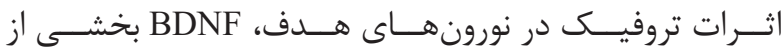

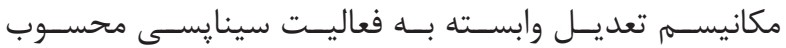

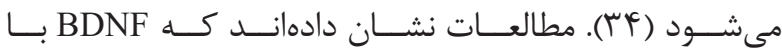

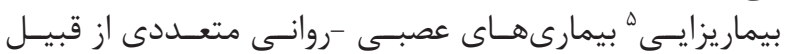

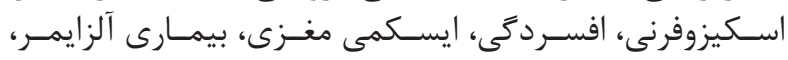

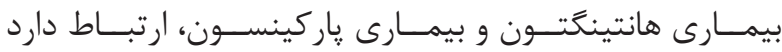

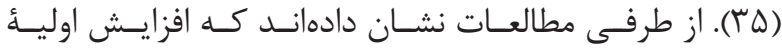

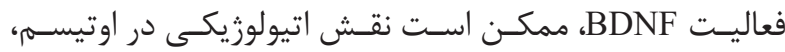

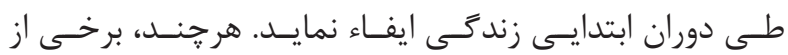

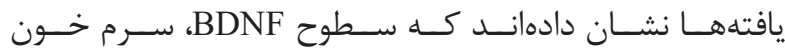

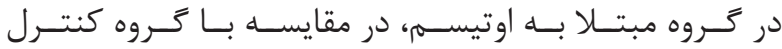

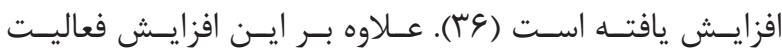

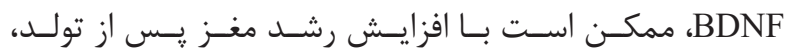

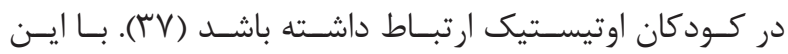

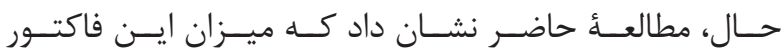

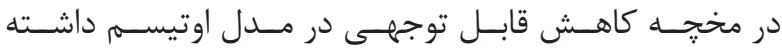

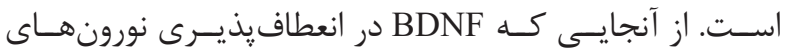

${ }^{5}$ Pathogenicity

${ }^{6}$ Protocatechuic acid

${ }^{7}$ Tyrosol
${ }^{8}$ Caftaric acid
${ }_{9}^{9}$ Glutathione peroxidase
${ }^{10}$ Malondialdehyde 
1. Shinoda Y, Sadakata T, Furuichi T. Animal models of autism spectrum disorder (ASD): a synaptic-level approach to autistic-like behavior in mice. Exp Anim. 2013; 62(2): 71-8.

2. Hastings PR, Brown T. Behavior problems of children with autism, parental self-efficacy, andmental health. Am J Ment Retard. 2002; 107(3): 222-32.

3. Deweerdt S. Mounting evidence implicates cerebellum in autism. SFARI. 2014; 8: 1-6.

4. Skefos J, Christopher C, Katelyn E, Holiday J, Weed $\mathrm{K}$, Levy E, et al. Regional alterations in purkinje cell density in patients with autism. PLoS One. 2014; 10: 1371. doi.org/10.1371/journal.pone.0081255.

5. Bergland Ch. How are purkinje cells in the cerebellum linked to autism? The Athlete's Way. 2014; 519: 14-51.

6. Miki T, Lee KY, Yokoyama T, Liu JQ, Kusaka T, Suzuki S, et al. Differential effects of neonatal maternal separation on the expression of neurotrophic factors in rat brain. II: regional differences in the cerebellum versus the cerebral cortex. Okajimas Folia Anat Jpn. 2013; 90(3): 53-8.

7. Choo M, Miyazaki T, Yamazaki M, Kawamura M, Nakazawa T, Zhang J, et al. Retrograde BDNF to TrkB signaling promotes synapse elimination in the developing cerebellum. Nat Commun. 2017; 8(1): 195. doi: $\quad 10.1038 / \mathrm{s} 41467-017-00260-w$.

8. Kim J, Lee S, Kang S, Kim SH, Kim JC, Yang $\mathrm{M}$, et al. Brain-derived neurotropic factor and GABAergic transmission in neurodegeneration and neurodegeneration. Neural Regen Res. 2017; 12(10): 1733-41.

9. Bath KG, Pimentel T. Effect of early postnatal exposure to valproate on neurobehavioral development and regional BDNF expression in two strains of mice. Epilepsy Behav. 2017; 70(Pt A): 110-7.

10. Rahmani F, Rezaei N. brain-derived neurotrophic factor role in autism remains elusive: a flashback on the route that has brought us here. Acta Med Iran. 2017; 55(12): 733-5.

11. Iwata K, Matsuzaki H, Takei N, ManabeT, Mori N. Animal models of autism: an epigenetic and environmental viewpoint. J Cent Nerv Syst Dis. 2010; 2: $37-44$.

12. Rinaldi T, Kulangara K, Antoniello K, Markram H. Elvated NMDA receptor levels and enhanced pos tsynaptic long-term potentiation induced by prenatal exposure to valproic acid. Proc Natl AcadSci USA. 2007; 104(33): 13501-6.

13. Kang J, Kim E. Suppression of NMDA receptor function in mice prenatally exposed to valproic acid improves social deficits and repetitive behaviors. Front Mol Neurosci. 2015; 8: 17. doi: 10.3389/ fnmol.2015.00017.

14. Nicolini C, Fahnestock M. The valproic acidinduced rodent model of autism. Exp Neurol. 2018; 299(Pt A): 217-27.

15. Khongrum J, Wattanathorn J. Laser acupuncture at HT7 improves the cerebellar disorders in valproic acidrat model of autism. J Acupunct Meridian Stud. 2017; 10(4): 231-9.

16. Rahimian S, Amin Yazdi A, Edalatmanesh MA. Environmental enrichment: a new therapy based on findings in animal models of autism. Shefaye Khatam. 2016; 4 (4) :89-98.

17. Yui K, Kawasaki Y, Yamada H, Ogawa S. Oxidative stress and nitric oxide in autism spectrum disorder and other neuropsychiatric disorders. CNS Neurol Disord Drug Targets. 2016; 15(5): 587-96.

18. Alasalvar C, Bolling BW. Review of nut phytochemicals, fat-soluble bioactives, antioxidant components and health effects. Br J Nutr. 2015; 113(2): S68-78.

19. Choubey S, Varughese LR, Kumar V, Beniwal V. Medicinal importance of gallic acid and its ester derivatives: a patent review. Pharm Pat Anal. 2015; 4(4): 305-15.

20. Sarjit A, Wang Y, Dykes GA. Antimicrobial activity of gallic acid against thermophilic Campylobacter is strain specific and associated with a loss of calcium ions. Food Microbiol. 2015; 46: 227-33.

21. Hsieh SC, Wu CC, Hsu SL, Yen JH. Molecular mechanisms of gallic acid-induced growth inhibition, apoptosis, and necrosis in hypertrophic scar fibroblasts. Life Sci. 179: 130-8.

22. Rafiei S, Bazyar Y, Edalatmanesh MA. Effect of gallic acid and endurance exercise training on bdnf in a model of hippocampal degeneration. Shefaye Khatam. 2016; 4(1) :1-6.

23. Garud MS, Kulkarni YA. Gallic acid attenuates type I diabetic nephropathy in rats. Chem Biol Interact. 2018; 
282: 69-76.

24. Sadoghi SD, Rahbariyan R. Investigation the effect of glycyrrhizic acid on ovarian follicle in polycystic ovarian syndrome mice model. SJIMU. 2017; 24(6): 138-48.

25. Edalatmanesh MA, Nikfarjam H, Moghadas M, Haddad-Mashadrizeh A, Robati R, Hashemzadeh MR. Histopathological and behavioral assessment of toxinproduced cerebellar lesion: a potent model for cell transplantation studies in the cerebellum. Cell J. 2014; 16(3): 325-34.

26. Sadoughi D, Khayatzadeh J. Effect of curcumin on hippocampal levels of brain-derived neurotrophic factor and serum levels of inflammatory cytokines in rat model for Alzheimer's disease. Shefaye Khatam. 2018; 6(1) :1-9.

27. Chris tensen J, Gronborg TK, Sorensen MJ, Schendel D, Parner ET, Pedersen LH. Prenatal valproate exposure and risk of autism spectrum disorders and childhood autism. JAMA. 2013; 309(16): 1696-703.

28. Edalatmanesh MA, Nikfarjam H, Vafaee F, Moghadas M. Increased hippocampal cell density and enhanced spatial memory in the valproic acid rat model of autism. Brain Res. 2013; 1526: 15-25.

29. Borzou Z, Edalatmanesh MA. The evaluation of brain derived neurotrophic factor and working memory in valproic acid animal model of autism. Shefaye Khatam. 2015; 3(4): 10-6.

30. Starowicz-Filip A, Chrobak AA, Moskała M, Krzyżewski RM, Kwinta B, Kwiatkowski S, et al. The role of the cerebellum in the regulation of language functions. Psychiatr Pol. 2017; 51(4): 661-71.

31. Teitelbaum P, Teitelbaum O, Fryman J, Maurer R. Infantile reflexes gone astray in autism. J Dev Learn Disord. 2000; 6: 15-22.

32. Mansouri MT, Farbood Y, Sameri JM, Sarkaki A,
Naghizadeh B, Rafieirad M. Neuroprotective effects of oral gallic acid against oxidative stress induced by 6-hydroxydopamine in rats. Food Chemistry. 2012; 138(2-3): 1028-33.

33. Nykjaer A, Willnow TE, Petersen CM. p75NTR--live or let die. Curr Opin Neurobiol. 2005; 15(1): 49-57.

34. Kishi T, Yoshimura R, Ikuta T, Iwata N. Brain-derived neurotrophic factor and major depressive disorder: evidence from meta-analyses. Front Psychiatry. 2018; 8: 308. doi: 10.3389/fpsyt.2017.00308.

35. Vazquez-Sanroman D, Sanchis-Segura C, Toledo R, Hernandez ME, Manzo J, Miquel M. The effects of enriched environment on BDNF expression in the mouse cerebellum depending on the length of exposure. Behav Brain Res. 2013; 243: 118-28.

36. Soori M, Edalatmanesh MA. Survey of serum levels of brain-derived neurotrophic factor in autistic rats. J Clin Res Paramed Sci. 2014; 3(4): 264-70.

37. Meng WD, Sun SJ, Yang J, Chu RX, Tu W, Liu Q. Elevated serum brain-derived neurotrophic factor (bdnf) but not bdnf gene val66met polymorphism is associated with autism spectrum disorders. Mol Neurobiol. 2017; 54(2): 1167-72.

38. Corona G, Vauzour D, Hercelin J, Williams CM, Spencer JP. Phenolic acid intake, delivered via moderate champagne wine consumption, improves spatial working memory via the modulation of hippocampal and cortical protein expression/activation. Antioxid Redox Signal. 2013; 19(14): 1676-89.

39. Doroodi L, Edalatmanesh MA. The histopathological evaluation of gallic acid on rat purkinje cells after trimethyltin intoxication. Shefaye Khatam. 2017; 5(2): 11-8.

40. Daglia M, Di Lorenzo A, Nabavi SF, Talas ZS, Nabavi SM. Polyphenols: well beyond the antioxidant capacity: gallic acid and related compounds as neuroprotective agents: you are what you eat! Curr Pharm Biotechnol. 2014; 15(4): 362-72. 